\title{
The speed of a biased random walk on a Galton-Watson tree is analytic*
}

\author{
Adam Bowditch $^{\dagger} \quad$ Yuki Tokushige ${ }^{\ddagger}$
}

\begin{abstract}
We prove that the speed of a biased random walk on a supercritical Galton-Watson tree conditioned to survive is analytic within the ballistic regime. This extends the previous work [12] in which it was shown that the speed is differentiable within the range of bias for which a central limit theorem holds.
\end{abstract}

Keywords: Galton-Watson tree; biased random walks; speed; analytic.

MSC2020 subject classifications: Primary 60J80, Secondary 60K37; 60J10; 05C81.

Submitted to ECP on June 4, 2020, final version accepted on August 17, 2020.

Supersedes arXiv: 2006.03433.

\section{Introduction}

The behaviour of biased random walks on Galton-Watson (GW) trees has been extensively studied since Lyons, Pemantle and Peres [16] proved the existence of a limiting speed. Since then, asymptotic properties of the walk have received considerable interest in the form of sub-ballistic escape rates [6], central limit theorems [10, 18], large deviations [13] and Einstein relations [8]. In this paper we are interested in the regularity properties of the speed for which there are many open problems both in this model (see [5]) and in the related models of biased random walks on percolation clusters [14] and random walk in random environment [19].

A novel feature of the model is that, even without leaves, monotonicity of the speed with respect to the bias (or offspring distribution) is non-trivial and remains an open problem except when the bias is sufficiently strong $[1,7,17]$. This can be attributed to the fact that certain sections of the tree will be exceptionally thin and the walk will typically move through them much slower than it would elsewhere. These adverse regions act as traps which may intensify as the bias away from the root is increased. With leaves, dead-ends form traps which create a similar slowing effect. The competing forces of the drift and the trapping result in a delicate relationship between the speed and the bias.

In this paper we study the speed of a biased random walk on a supercritical GW tree (with or without leaves) as a function of the bias. We prove that the speed is analytic within the range of bias such that the speed is strictly positive. This builds on [12] where it has been shown that the speed is differentiable and an expression for the derivative

${ }^{*}$ Retraction note: https://doi.org/10.1214/ECP437.

${ }^{\dagger}$ National University of Singapore, Department of Mathematics, Singapore. E-mail: matamb@nus .edu.sg

${ }^{\ddagger}$ Kyoto University, RIMS, Kyoto 606-8502, Japan. E-mail: tokusige@kurims.kyoto-u.ac.jp 


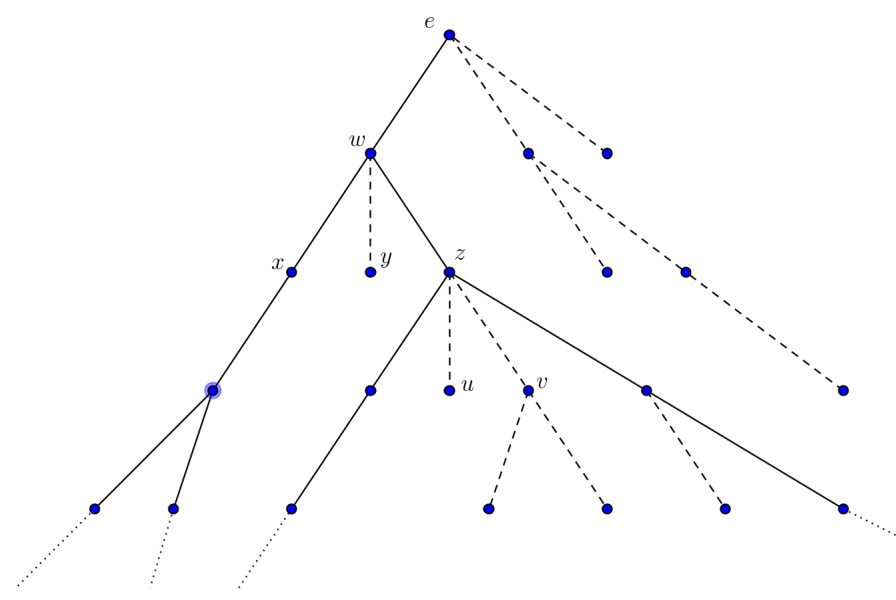

Figure 1: A sample section of a supercritical GW-tree conditioned to survive $\mathbf{T}$ with solid lines representing the backbone $\mathbf{T}_{g}$ and dashed lines representing the traps. Here, the root $e$ is the parent of $w$ (i.e. $e=\pi(w)$ ) which has children $x, y, z$ where $x, z$ are on the backbone and $y$ is a bud in the only trap rooted at $w$. Similarly, $u, v$ are two of the children of $z$, both of which are buds of individual traps rooted at

was given in terms of the covariance of a certain 2-dimensional Gaussian. For biased random walks on subcritical GW trees conditioned to survive, an explicit expression for the speed has been obtained in [11] which is analytic within the strictly positive speed regime.

We briefly describe the supercritical GW-tree conditioned on survival via the Harris decomposition; for more detail see $[3,15]$. Let $\left\{p_{k}\right\}_{k \geq 0}$ denote the offspring distribution of a GW-process $W_{n}$ with a single progenitor, mean $\mu>1$ and probability generating function $f$. The process $W_{n}$ gives rise to a random tree $\mathbf{T}_{f}$ where individuals are represented by vertices and edges connect individuals with their offspring. Let $q$ denote the extinction probability of $W_{n}$ which is strictly less than 1 since $\mu>1$ and non-zero only when $p_{0}>0$. In this case we then define

$$
g(s):=\frac{f((1-q) s+q)-q}{1-q} \quad \text { and } \quad h(s):=\frac{f(q s)}{q}
$$

which are generating functions of a GW-process without deaths and a subcritical GWprocess respectively (cf. [3, Chapter I.12]). An $f$-GW-tree conditioned on nonextinction $\mathbf{T}$ can be constructed by first generating a $g$-GW-tree $\mathbf{T}_{g}$ and then, to each vertex $x$ of $\mathbf{T}_{g}$, appending a random number of independent $h$-GW-trees (see Figure 1 ). We refer to $\mathbf{T}_{g}$ as the backbone of $\mathbf{T}$, the finite trees appended to $\mathbf{T}_{g}$ as the traps and the vertices in the first generation of the traps as the buds.

We now introduce the biased random walk on a fixed tree $\mathscr{T}$. We denote by $e(\mathscr{T})$ the root, which is the vertex representing the unique progenitor. For $x \in \mathscr{T}$, let $\pi(x)$ denote the parent of $x$ and $\nu(x)$ the number of children of $x$. A $\lambda$-biased random walk on $\mathscr{T}$ is a random walk $\left(Z_{n}\right)_{n \geq 0}$ on the vertices of $\mathscr{T}$ started from $e(\mathscr{T})$ with transition probabilities

$$
P_{\lambda}^{\mathscr{T}}\left(Z_{n+1}=y \mid Z_{n}=x\right):= \begin{cases}\frac{\lambda}{\lambda+\nu(x)}, & \text { if } y=\pi(x) \\ \frac{1}{\lambda+\nu(x)}, & \text { if } x=\pi(y) \neq e(\mathscr{T}), \\ \frac{1}{\nu(x)}, & \text { if } x=\pi(y)=e(\mathscr{T}) \\ 0, & \text { otherwise. }\end{cases}
$$


The speed of a biased random walk on a Galton-Watson tree is analytic

For $x \in \mathscr{T}$ we then write $P_{\lambda, x}^{\mathscr{T}}(\cdot):=P_{\lambda}^{\mathscr{T}}\left(\cdot \mid Z_{0}=x\right)$ for the law of the walk on $\mathscr{T}$ started from $x$. We use $P_{\lambda}(\cdot):=\int P_{\lambda}^{\mathbf{T}}(\cdot) \mathbb{P}(\mathrm{dT})$ for the annealed law obtained by averaging the quenched law $P_{\lambda}^{\mathbf{T}}$ with respect to the law $\mathbb{P}$ on $f$-GW-trees conditioned to survive and denote the expectation with respect to $P_{\lambda}$ (resp. $P_{\lambda}^{\mathscr{T}}$ ) by $E_{\lambda}$ (resp. $E_{\lambda}^{\mathscr{T}}$ ).

For $x \in \mathbf{T}$, let $d(x)$ denote the distance between $x$ and the root of the tree and write

$$
\lambda_{c}:= \begin{cases}0 & \text { if } p_{0}=0 \\ f^{\prime}(q) & \text { if } p_{0}>0 .\end{cases}
$$

In [16], Lyons, Pemantle and Peres showed that if $\lambda \in\left(\lambda_{c}, \mu\right)$ then the walk is ballistic; that is, $d\left(Z_{n}\right) n^{-1}$ converges $P_{\lambda}$-a.s. to a deterministic constant $v_{\lambda}>0$ called the speed of the walk. When $\lambda \geq \mu$ the walk is recurrent and $d\left(Z_{n}\right) n^{-1}$ converges $P_{\lambda}$-a.s. to 0 . When $\lambda$ is small and $p_{0}>0$, the walk is transient but slowed by having to make long sequences of movements against the bias in order to escape the traps; in particular, if $\lambda \leq \lambda_{c}$ then the slowing effect is strong enough to cause $d\left(Z_{n}\right) n^{-1}$ to converge $P_{\lambda}$-a.s. to 0 . This regime has been studied further in [6] and [9] where polynomial scaling results are shown.

The aim of this paper is to study how the value of $v_{\lambda}$ depends on the parameter of bias $\lambda$; specifically, our main result is the following.

Theorem 1.1. The function $\lambda \mapsto v_{\lambda}$ is analytic on $\left(\lambda_{c}, \mu\right)$

We now introduce a result shown in [2] which will play an important role in this paper. Let $\left(\mathbf{T}_{+, i}\right)_{i \geq 1}$ be independent random trees which have the law of $\mathbf{T}_{f}$ (and are also independent of $\mathbf{T}$ ). We will denote by $\mathscr{T}^{*}$ a new tree obtained by adding to the graph $\mathscr{T}$ an edge connecting $e(\mathscr{T})$ and a new vertex $e^{*}(\mathscr{T})$. For $x \in \mathscr{T}^{*}$, define the first return time $\sigma_{x}$ by $\sigma_{x}:=\inf \left\{n \geq 1 ; Z_{n}=x\right\}$. We then define

$$
\beta:=P_{\lambda}^{\mathbf{T}^{*}}\left(\sigma_{e^{*}}=\infty\right) \text { and } \beta_{+, i}:=P_{\lambda}^{\mathbf{T}_{+, i}^{*}}\left(\sigma_{e^{*}}=\infty\right)
$$

We note that these random variables depend on the bias $\lambda$; however, we omit this from the notation for simplicity since we never include these at varying values of $\lambda$ in the same equation. In [2], Aïdekon showed that the speed can be expressed as

$$
v_{\lambda}=\mathbb{E}\left[\frac{(\nu-\lambda) \beta}{\lambda-1+\beta+\sum_{i=1}^{\nu} \beta_{+, i}}\right] / \mathbb{E}\left[\frac{(\nu+\lambda) \beta}{\lambda-1+\beta+\sum_{i=1}^{\nu} \beta_{+, i}}\right] .
$$

The variables $\beta$ and $\left(\beta_{+, i}\right)_{i \geq 1}$ are independent of each other and also of $\nu$. When the tree $\mathbf{T}_{+, i}$ is finite we have that $\beta_{+, i}=0$ whereas, conditioned on $\mathbf{T}_{+, i}$ being infinite, we have that $\beta_{+, i}$ is equal in distribution to $\beta$. In particular, since each of the trees $\mathbf{T}_{+, i}$ is infinite independently with probability $1-q$ we can rewrite (1.1) as

$$
v_{\lambda}=\mathbb{E}\left[\frac{(\nu-\lambda) \beta_{0}}{\lambda-1+\sum_{i=0}^{\nu_{q}} \beta_{i}}\right] / \mathbb{E}\left[\frac{(\nu+\lambda) \beta_{0}}{\lambda-1+\sum_{i=0}^{\nu_{q}} \beta_{i}}\right]
$$

where

$$
\beta_{i}:=P_{\lambda}^{\mathbf{T}_{i}^{*}}\left(\sigma_{e^{*}}=\infty\right)
$$

for independent trees $\left(\mathbf{T}_{i}\right)_{i \geq 0}$ with the law of $\mathbf{T}$ and $\nu_{q}$ has a binomial distribution with $\nu$ trials of success probability $1-q$.

We summarise below three properties of non-return probabilities which will be frequently utilised in this paper. Firstly, the variables $\beta, \beta_{i}$ and $\beta_{+, i}$ are all $\mathbb{P}$-almost surely monotonically decreasing in $\lambda$. This fact can be easily seen by Rayleigh's monotonicity principle and Theorem 2.11 in [4]. Secondly, the distribution of $\beta_{0}$ is same as that of $P_{\lambda}^{\mathbf{T}_{g}^{*}}\left(\sigma_{e^{*}}=\infty\right)$ since traps appended to the backbone do not affect the occurrence of the event $\left\{\sigma_{e^{*}}=\infty\right\}$. Finally, $\beta_{i} \geq 1-\lambda$ by a coupling with a biased random walk on $\mathbb{Z}_{+}$. 
The remainder of the paper is devoted to proving Theorem 1.1. We split the proof into two parts; in Section 2 we study the return probability $\beta$ and prove several technical estimates then, in Section 3, we approximate the speed by a sequence of analytic functions and show that this sequence converges compactly.

\section{Return times}

We first prove several technical results that will be useful throughout the proof of Theorem 1.1. Let us note that, since $\left(\beta_{i}\right)_{i \geq 0}$ are i.i.d. and independent of $\nu$ and $\nu_{q}$,

$$
\begin{aligned}
\mathbb{E}\left[\frac{(\nu \pm \lambda) \beta_{0}}{\lambda-1+\sum_{i=0}^{\nu_{q}} \beta_{i}}\right] & =\mathbb{E}\left[\frac{(\nu \pm \lambda) \sum_{i=0}^{\nu_{q}} \beta_{i}}{\left(\nu_{q}+1\right)\left(\lambda-1+\sum_{i=0}^{\nu_{q}} \beta_{i}\right)}\right] \\
& =\mathbb{E}\left[\frac{\nu \pm \lambda}{\nu_{q}+1}\right]-\mathbb{E}\left[\frac{(\nu \pm \lambda)(\lambda-1)}{\left(\nu_{q}+1\right)\left(\lambda-1+\sum_{i=0}^{\nu_{q}} \beta_{i}\right)}\right] .
\end{aligned}
$$

Using that $\mathbb{E}\left[\frac{\nu \pm \lambda}{\nu_{q}+1}\right]$ in (2.1) is analytic, in order to prove Theorem 1.1 it suffices to prove that

$$
\mathbb{E}\left[\frac{(\nu-\lambda)(\lambda-1)}{\left(\nu_{q}+1\right)\left(\lambda-1+\sum_{i=0}^{\nu_{q}} \beta_{i}\right)}\right], \quad \mathbb{E}\left[\frac{(\nu+\lambda)(\lambda-1)}{\left(\nu_{q}+1\right)\left(\lambda-1+\sum_{i=0}^{\nu_{q}} \beta_{i}\right)}\right]
$$

are analytic. We restrict ourselves to showing analyticity of the second term in a fixed interval $[a, b] \subset\left(\lambda_{c}, \mu\right)$ (without loss of generality we assume that $a<1<b$ ). The first term follows by an identical argument and, since this holds for any such $[a, b]$, analyticity on $\left(\lambda_{c}, \mu\right)$ follows.

Note that, for $\lambda \geq 1$ we have that

$$
\mathbb{E}\left[\frac{(\nu+\lambda) \sum_{i=0}^{\nu_{q}} \beta_{i}}{\left(\nu_{q}+1\right)\left(\lambda-1+\sum_{i=0}^{\nu_{q}} \beta_{i}\right)}\right] \geq \mathbb{E}\left[\frac{\sum_{i=0}^{\nu_{q}} \beta_{i}}{\mu+\nu_{q}}\right] \geq \mu^{-1} \mathbb{P}\left(\nu_{q}=0\right) \mathbb{E}\left[\beta_{0}\right]>0 .
$$

By the monotonicity of $\beta_{0}$ in $\lambda$, we obtain that the denominator in (1.2) is uniformly bounded below in any compact interval contained in $[1, \mu)$. For $\lambda \leq 1$

$$
\mathbb{E}\left[\frac{(\nu+\lambda) \sum_{i=0}^{\nu_{q}} \beta_{i}}{\left(\nu_{q}+1\right)\left(\lambda-1+\sum_{i=0}^{\nu_{q}} \beta_{i}\right)}\right] \geq \frac{\mathbb{P}\left(\nu_{q} \geq 1\right)}{2} \mathbb{E}\left[\frac{\sum_{i=0}^{\nu_{q}} \beta_{i}}{\lambda-1+\sum_{i=0}^{\nu_{q}} \beta_{i}} \mid \nu_{q} \geq 1\right] \geq \frac{\mathbb{P}\left(\nu_{q} \geq 1\right)}{2}>0
$$

since $\beta_{0} \geq 1-\lambda$. We therefore have that the denominator in (1.2) is uniformly bounded below in any compact interval contained in $\left(\lambda_{c}, \mu\right)$.

We next prove a technical lemma that will be used throughout to deal with the case when $\nu_{q}=0$, which occurs only when $p_{0}>0$. This case typically causes additional difficulty because when $\nu_{q}=0$ and $\lambda<1$ we do not have a lower bound on $\lambda-1+\sum_{i=0}^{\nu_{q}} \beta_{i}$ which is standard when $\nu_{q} \geq 1$ because $\beta_{0}, \beta_{1} \geq 1-\lambda$.

Lemma 2.1. Suppose that $p_{0} \neq 0$ and $[a, b] \subset\left(\lambda_{c}, \mu\right)$ with $a<1$. For any $1<p<$ $\log \left(\lambda_{c}\right) / \log (a)$ we have that

$$
\sup _{\lambda \in[a, b]} \mathbb{E}\left[\left(\frac{|\lambda-1|}{\lambda-1+\beta_{0}}\right)^{p}\right]<\infty .
$$

Proof. For $\lambda \geq 1$ we have that

$$
\frac{|\lambda-1|}{\lambda-1+\beta_{0}} \leq 1
$$

and for $\lambda<1$ we have that $\beta_{0} \geq 1-\lambda$ therefore

$$
\frac{|\lambda-1|}{\lambda-1+\beta_{0}} \leq \frac{\beta_{0}}{\lambda-1+\beta_{0}} \text {. }
$$


The speed of a biased random walk on a Galton-Watson tree is analytic

It therefore suffices to show that

$$
\sup _{\lambda \in[a, 1)} \mathbb{E}\left[\left(\frac{\beta_{0}}{\lambda-1+\beta_{0}}\right)^{p}\right]<\infty .
$$

For $x \in \mathbf{T}_{g}$ write $\beta(x):=P_{\lambda, x}^{\mathbf{T}_{g}^{*}}\left(\sigma_{\pi(x)}=\infty\right)$ and $c(x):=\left\{y \in \mathbf{T}_{g}^{*}: \pi(y)=x\right\}$ for the set of children of $x$. By [2, (4.2)] we have that

$$
\beta_{0}=\beta(e)=\frac{\sum_{y \in c(e)} \beta(y)}{\lambda+\sum_{y \in c(e)} \beta(y)}
$$

and thus

$$
\frac{\beta_{0}}{\lambda-1+\beta_{0}}=\frac{1}{\lambda} \cdot \frac{\sum_{y \in c(e)} \beta(y)}{\lambda-1+\sum_{y \in c(e)} \beta(y)} .
$$

Using that $\beta(x) \geq 1-\lambda$ for any $x$ we have that if $\nu(x) \geq 2$ then

$$
\frac{\sum_{y \in c(x)} \beta(y)}{\lambda-1+\sum_{y \in c(x)} \beta(y)} \leq \frac{\sum_{y \in c(x)} \beta(y)}{\sum_{y \in c(x)} \beta(y)-\min _{z \in c(x)} \beta(z)} \leq 2 .
$$

Let $W_{n}^{g}$ denote the $n^{\text {th }}$ generation size of $\mathbf{T}_{g}^{*}$ starting from $W_{0}^{g}=1$ representing $e$. Write $\mathcal{C}_{j}:=\left\{W_{n}^{g}=1 \forall n \leq j\right\}$ for the event that the first $j$ generations have only a single vertex and on this event let $x_{j}$ denote that vertex. Repeatedly applying (2.3), we have that

$$
\frac{\beta_{0}}{\lambda-1+\beta_{0}} \leq \lambda^{-j} \frac{\beta\left(x_{j}\right)}{\lambda-1+\beta\left(x_{j}\right)} \mathbf{1}_{\mathcal{C}_{j}}+2 \sum_{i=0}^{j-1} \lambda^{-i} \mathbf{1}_{\mathcal{C}_{i+1}^{c} \cap \mathcal{C}_{i}} .
$$

Since $p_{0} \neq 0$ we have that, for $n \geq 0$,

$$
\mathbb{P}\left(W_{n+1}^{g}=1 \mid W_{n}^{g}=1\right)=g^{\prime}(0)=f^{\prime}(q)=\lambda_{c} .
$$

Therefore, for $t>0$, we have that

$$
\begin{aligned}
\mathbb{P}\left(\frac{\beta_{0}}{\lambda-1+\beta_{0}}>t\right) & \leq \mathbb{P}\left(\lambda^{-j} \frac{\beta\left(x_{j}\right)}{\lambda-1+\beta\left(x_{j}\right)} \mathbf{1}_{\mathcal{C}_{j}}+2 \sum_{i=1}^{j-1} \lambda^{-i} \mathbf{1}_{\mathcal{C}_{i+1}^{c} \cap \mathcal{C}_{i}}>t\right) \\
& \leq \mathbb{P}\left(\mathcal{C}_{\log (t / 2) / \log \left(\lambda^{-1}\right)}\right) \\
& \leq \lambda_{c}^{\left\lfloor\frac{\log (t / 2)}{\log \left(\lambda^{-1}\right)}\right\rfloor .}
\end{aligned}
$$

Since $\lambda_{c}<a<1$ we have that, for $p \in\left[1, \log \left(\lambda_{c}\right) / \log (a)\right]$,

$$
\sup _{\lambda \in[a, 1)} \mathbb{P}\left(\frac{\beta_{0}}{\lambda-1+\beta_{0}}>t\right) \leq C t^{-p}
$$

which proves that (2.2) holds.

Let $\mathbf{T}_{g, i}^{*}$ denote the backbone of $\mathbf{T}_{i}^{*}$ and write

$$
\beta_{i, n}:=P_{\lambda}^{\mathbf{T}_{g, i}^{*}}\left(\sigma_{e^{*}}>n\right)=\beta_{i}+P_{\lambda}^{\mathbf{T}_{g, i}^{*}}\left(n<\sigma_{e^{*}}<\infty\right)
$$

for the probability that the first return to $e^{*}$ in $\mathbf{T}_{g, i}^{*}$ occurs after time $n$. Note that, by restricting to the backbone, the time spent in the finite traps in $\mathbf{T}_{i}^{*}$ does not contribute to $\sigma_{e^{*}}$. Moreover, $\mathbf{T}_{g, i}^{*}$ is a GW tree whose offspring distribution has no deaths and mean $\mu$. By [12, Lemma 4.7], for any $[a, b] \subset(0, \mu)$ we have that

$$
\lim _{n \rightarrow \infty} \sup _{\lambda \in[a, b]} \mathbb{E}\left[P_{\lambda}^{\mathbf{T}_{g}^{*}}\left(n<\sigma_{e^{*}}<\infty\right)\right]=0
$$


The speed of a biased random walk on a Galton-Watson tree is analytic

Using (2.4) and Lemma 2.1, we now prove that the convergence in probability of $|\lambda-1| /\left(\lambda-1+\beta_{0, n}\right)$ is uniform within compact intervals of $\left(\lambda_{c}, \mu\right)$. Similarly to Lemma 2.1, this will be important when studying the case when $\nu_{q}=0$.

Lemma 2.2. Suppose that $p_{0} \neq 0$ and $[a, b] \subset\left(\lambda_{c}, \mu\right)$. For any $\varepsilon>0$ we have that

$$
\lim _{n \rightarrow \infty} \sup _{\lambda \in[a, b]} \mathbb{P}\left(\frac{|\lambda-1|}{\lambda-1+\beta_{0}}-\frac{|\lambda-1|}{\lambda-1+\beta_{0, n}}>\varepsilon\right)=0
$$

Proof. We split into two cases. First, for $\lambda \in[1, b]$ we have that

$$
0 \leq \frac{|\lambda-1|}{\lambda-1+\beta_{0}}-\frac{|\lambda-1|}{\lambda-1+\beta_{0, n}}=\frac{|\lambda-1|\left(\beta_{0, n}-\beta_{0}\right)}{\left(\lambda-1+\beta_{0}\right)\left(\lambda-1+\beta_{0, n}\right)} \leq \frac{\beta_{0, n}-\beta_{0}}{\beta_{0}+\beta_{0, n}} \leq 1 .
$$

Therefore, for any $\varepsilon, \vartheta>0$ we have that

$$
\begin{aligned}
& \sup _{\lambda \in[1, b]} \mathbb{P}\left(\frac{|\lambda-1|}{\lambda-1+\beta_{0}}-\frac{|\lambda-1|}{\lambda-1+\beta_{0, n}}>\varepsilon\right) \\
& \leq \sup _{\lambda \in[1, b]} \mathbb{P}\left(\frac{\beta_{0, n}-\beta_{0}}{\beta_{0}+\beta_{0, n}}>\varepsilon\right) \\
& \leq \sup _{\lambda \in[1, b]} \mathbb{P}\left(\beta_{0, n}-\beta_{0}>\varepsilon \vartheta\right)+\sup _{\lambda \in[1, b]} \mathbb{P}\left(\beta_{0}+\beta_{0, n}<\vartheta\right) \\
& \leq \frac{1}{\varepsilon \vartheta} \sup _{\lambda \in[1, b]} \mathbb{E}\left[\beta_{0, n}-\beta_{0}\right]+\mathbb{P}\left(P_{b}^{\mathbf{T}_{g}^{*}}\left(\sigma_{e^{*}}=\infty\right)<\vartheta\right)
\end{aligned}
$$

since $\beta_{0}$ is monotonically decreasing in $\lambda$ and $\beta_{0, n} \geq 0$. By (2.4) we have that

$$
\sup _{\lambda \in[1, b]} \mathbb{E}\left[\beta_{0, n}-\beta_{0}\right]=\sup _{\lambda \in[1, b]} \mathbb{E}\left[P_{\lambda}^{\mathbf{T}_{g}^{*}}\left(n<\sigma_{e^{*}}<\infty\right)\right]
$$

converges to 0 as $n \rightarrow \infty$. Since $b<\mu$ we have that the walk with bias $\lambda=b$ is $\mathbb{P}$-a.s. transient therefore $\mathbb{P}\left(P_{b}^{\mathbf{T}_{g}^{*}}\left(\sigma_{e^{*}}=\infty\right)<\vartheta\right)$ converges to 0 as $\vartheta \rightarrow 0$. It follows that (2.5) converges to 0 as $n \rightarrow \infty$

We now consider $\lambda \in[a, 1]$. Since the walk with bias $\lambda=1$ is $\mathbb{P}$-a.s. transient and $\beta_{0}$ is monotonically decreasing in $\lambda$ we have that for any $\eta>0$ there exists $\vartheta_{\eta}, \delta_{\eta}>0$ such that

$$
\sup _{\lambda \in\left[1-\delta_{\eta}, 1\right]} \mathbb{P}\left(\lambda-1+\beta_{0} \leq \vartheta_{\eta}\right) \leq \mathbb{P}\left(P_{1}^{\mathbf{T}_{g}^{*}}\left(\sigma_{e^{*}}=\infty\right) \leq \vartheta_{\eta}+\delta_{\eta}\right) \leq \eta
$$

Using this with (2.4) and Markov's inequality we then have that

$$
\begin{aligned}
& \lim _{n \rightarrow \infty} \sup _{\lambda \in\left[1-\delta_{\eta}, 1\right]} \mathbb{P}\left(\frac{|\lambda-1|}{\lambda-1+\beta_{0}}-\frac{|\lambda-1|}{\lambda-1+\beta_{0, n}}>\varepsilon\right) \\
& \leq \lim _{n \rightarrow \infty} \sup _{\lambda \in\left[1-\delta_{\eta}, 1\right]} \mathbb{P}\left(\frac{(1-\lambda)\left(\beta_{0, n}-\beta_{0}\right)}{\left(\lambda-1+\beta_{0}\right)^{2}}>\varepsilon\right) \\
& \leq \eta+\frac{1}{\varepsilon \vartheta_{\eta}^{2}} \lim _{n \rightarrow \infty} \sup _{\lambda \in\left[1-\delta_{\eta}, 1\right]} \mathbb{E}\left[\beta_{0, n}-\beta_{0}\right] \\
& \leq \eta .
\end{aligned}
$$

Fix $p \in\left(1, \log \left(\lambda_{c}\right) / \log (a)\right)$ and write $\mathcal{B}_{\eta, \lambda}:=\left\{\lambda-1+\beta_{0}>\eta^{1 / p}(1-\lambda)\right\}$. Then, by Markov's inequality and Lemma 2.1,

$$
\sup _{\lambda \in\left[a, 1-\delta_{\eta}\right]} \mathbb{P}\left(\mathcal{B}_{\eta, \lambda}^{c}\right) \leq \eta \sup _{\lambda \in[a, 1]} \mathbb{E}\left[\left(\frac{|\lambda-1|}{\lambda-1+\beta_{0}}\right)^{p}\right] \leq C \eta
$$


Recalling that $\beta_{0, n} \geq \beta_{0}$ we have that

$$
\begin{aligned}
& \sup _{\lambda \in\left[a, 1-\delta_{\eta}\right]} \mathbb{P}\left(\frac{|\lambda-1|}{\lambda-1+\beta_{0}}-\frac{|\lambda-1|}{\lambda-1+\beta_{0, n}}>\varepsilon, \mathcal{B}_{\eta, \lambda}\right) \\
\leq & \sup _{\lambda \in\left[a, 1-\delta_{\eta}\right]} \mathbb{P}\left(\frac{(1-\lambda)\left(\beta_{0, n}-\beta_{0}\right) \mathbf{1}_{\mathcal{B}_{\eta, \lambda}}}{\left(\lambda-1+\beta_{0}\right)^{2}}>\varepsilon\right) \\
\leq & \varepsilon^{-1} \sup _{\lambda \in\left[a, 1-\delta_{\eta}\right]} \mathbb{E}\left[\frac{(1-\lambda)\left(\beta_{0, n}-\beta_{0}\right) \mathbf{1}_{\mathcal{B}_{\eta, \lambda}}}{\left(\lambda-1+\beta_{0}\right)^{2}}\right] \\
\leq & \frac{1}{\varepsilon \eta^{2 / p} \delta_{\eta}} \sup _{\lambda \in[a, 1]} \mathbb{E}\left[\beta_{0, n}-\beta_{0}\right]
\end{aligned}
$$

which converges to 0 as $n \rightarrow \infty$ by (2.4). Combining with (2.6) and (2.7) we have that

$$
\lim _{n \rightarrow \infty} \sup _{\lambda \in[a, 1]} \mathbb{P}\left(\frac{|\lambda-1|}{\lambda-1+\beta_{0}}-\frac{|\lambda-1|}{\lambda-1+\beta_{0, n}}>\varepsilon\right) \leq C \eta
$$

which completes the proof since $\eta>0$ was arbitrary.

\section{Approximations of the speed}

We now show that we can approximate $v_{\lambda}$ by a sequence of analytic functions which converge compactly to the speed. Our approximation is formed by replacing the random variables $\beta_{i}$ with the approximations $\beta_{i, n}$. We first show that this approximation is analytic.

Lemma 3.1. For any $n \geq 1$

$$
\lambda \mapsto \mathbb{E}\left[\frac{(\nu+\lambda)(\lambda-1)}{\left(\nu_{q}+1\right)\left(\lambda-1+\sum_{i=0}^{\nu_{q}} \beta_{i, n}\right)}\right]
$$

is analytic on $\left(\lambda_{c}, \mu\right)$.

Proof. For a tree $\mathscr{T}$ write $\mathscr{T}[n]$ for the truncation of $\mathscr{T}$ up to level $n$ and define

$$
\mathcal{A}_{m, n}:=\{\nu \leq m\} \cap \bigcap_{i=0}^{\nu_{q}}\left\{\max \left\{\nu(x): x \in \mathbf{T}_{i}^{*}[n]\right\} \leq m\right\}
$$

to be the event that every vertex in some $\mathbf{T}_{i}^{*}$ for $i=1, \ldots, \nu_{q}$ up to level $n$ has degree at most $m$ and also that $\nu \leq m$.

For $\nu \leq m$ there are only finitely many $\nu_{q}$ and families of trees $\left\{\mathbf{T}_{i}^{*}[n]\right\}_{i=0}^{\nu_{q}}$ which satisfy $\mathcal{A}_{m, n}$. Since each $\beta_{i, n}$ is an analytic function which only depends on the first $n$ steps of the walk we therefore have that

$$
\mathbb{E}\left[\frac{(\nu+\lambda)(\lambda-1) \mathbf{1}_{\mathcal{A}_{m, n}}}{\left(\nu_{q}+1\right)\left(\lambda-1+\sum_{i=0}^{\nu_{q}} \beta_{i, n}\right)}\right]
$$

is analytic. We wish to show that the expectation in (3.2) converges compactly to (3.1) as $m \rightarrow \infty$.

We first show that $\lim _{m \rightarrow \infty} \mathbb{P}\left(\mathcal{A}_{m, n}^{c}\right)=0$. On the event $\mathcal{A}_{m, n}^{c}$ we must have that

$$
\nu+\sum_{i=0}^{\nu_{q}}\left|\mathbf{T}_{i}^{*}[n]\right|>m
$$


The speed of a biased random walk on a Galton-Watson tree is analytic

By Markov's inequality it therefore follows that $\mathbb{P}\left(\mathcal{A}_{m, n}^{c}\right)$ is bounded above by

$$
m^{-1} \mathbb{E}\left[\nu+\sum_{i=0}^{\nu_{q}}\left|\mathbf{T}_{i}^{*}[n]\right|\right] \leq m^{-1} \mathbb{E}[\nu+1]\left(1+\sum_{k=0}^{n} \mathbb{E}\left[W_{k}\right]\right) \leq m^{-1} \mathbb{E}[\nu+1]\left(1+\sum_{k=0}^{n} \mu^{k}\right)
$$

which converges to 0 as $m \rightarrow \infty$.

Both (3.1) and (3.2) are equal to 0 when $\lambda=1$ therefore we exclude this case and write $\Lambda:=[a, b] \backslash\{1\}$. Note that for $\lambda>1$ we have

$$
\left|\frac{(\nu+\lambda)(\lambda-1)}{\left(\nu_{q}+1\right)\left(\lambda-1+\sum_{i=0}^{\nu_{q}} \beta_{i, n}\right)}\right| \leq \frac{\nu+\lambda}{\nu_{q}+1} \leq \nu+\lambda .
$$

For $\lambda<1$ and $\nu_{q} \geq 1$ we have

$$
\left|\frac{(\nu+\lambda)(\lambda-1)}{\left(\nu_{q}+1\right)\left(\lambda-1+\sum_{i=0}^{\nu_{q}} \beta_{i, n}\right)}\right| \leq \frac{(\nu+\lambda)(1-\lambda)}{\left(\nu_{q}+1\right) \sum_{i=1}^{\nu_{q}} \beta_{i, n}} \leq \nu+\lambda
$$

since $\beta_{i, n} \geq 1-\lambda$. It therefore follows that

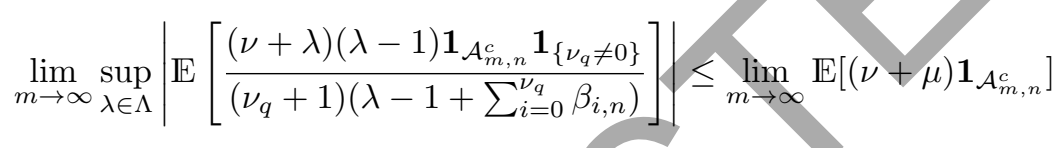

which converges to 0 as $m \rightarrow \infty$ by dominated converge since $\lim _{m \rightarrow \infty} \mathbb{P}\left(\mathcal{A}_{m, n}^{c}\right)=0$.

For the case where $\lambda<1$ and $\nu_{q}=0$, using the Cauchy-Schwarz inequality and independence of $\nu$ and $\nu_{q}$ with $\beta_{0}$ we have that

$$
\begin{aligned}
& \sup _{\lambda \in \Lambda}\left|\mathbb{E}\left[\frac{(\nu+\lambda)(\lambda-1) \mathbf{1}_{\mathcal{A}_{m, n}^{c}} \mathbf{1}_{\left\{\nu_{q}=0\right\}}}{\lambda-1+\beta_{0, n}}\right]\right| \\
& \leq \mathbb{P}\left(\mathcal{A}_{m, n}^{c}\right)^{\frac{p-1}{p}} \mathbb{E}\left[(\nu+\mu)^{p} \mathbf{1}_{\left\{\nu_{q}=0\right\}}\right]^{\frac{1}{p}} \sup _{\lambda \in \Lambda} \mathbb{E}\left[\left(\frac{|\lambda-1|}{\lambda-1+\beta_{0}}\right)^{p}\right]^{\frac{1}{p}} .
\end{aligned}
$$

If $p_{0}=0$ then we cannot have that $\nu_{q}=0$ therefore we may assume that $p_{0} \neq 0$. Therefore, by Lemma 2.1, we have that

$$
\sup _{\lambda \in \Lambda} \mathbb{E}\left[\left(\frac{|\lambda-1|}{\lambda-1+\beta_{0}}\right)^{p}\right]^{\frac{1}{p}}<\infty
$$

for $p>1$ sufficiently close to 1 . Moreover, since $p_{0} \neq 0$ we have that $q \in(0,1)$ and therefore

$$
\mathbb{E}\left[(\nu+\mu)^{p} \mathbf{1}_{\left\{\nu_{q}=0\right\}}\right]^{\frac{1}{p}}=\sum_{k=0}^{\infty}(k+\mu)^{p} \mathbb{P}(\nu=k) q^{k}<\infty .
$$

We therefore have that (3.3) converges to 0 as $m \rightarrow \infty$ which completes the proof.

We now prove Theorem 1.1 by showing that our analytic approximation converges compactly to the speed.

Proof of Theorem 1.1. To show analyticity of $v_{\lambda}$ on the interval $[a, b]$ it remains to show that

$$
\lim _{n \rightarrow \infty} \sup _{\lambda \in[a, b]} \mathbb{E}\left[\frac{(\nu+\lambda)|\lambda-1|}{\left(\nu_{q}+1\right)\left(\lambda-1+\sum_{i=0}^{\nu_{q}} \beta_{i}\right)}-\frac{(\nu+\lambda)|\lambda-1|}{\left(\nu_{q}+1\right)\left(\lambda-1+\sum_{i=0}^{\nu_{q}} \beta_{i, n}\right)}\right]=0 .
$$


The speed of a biased random walk on a Galton-Watson tree is analytic

To begin, let us consider the case $p_{0}=0$ which implies that $\nu_{q}=\nu \geq 1$. For $\varepsilon>0$, write $\mathcal{B}_{\varepsilon}:=\left\{\sum_{i=1}^{\nu_{q}} \beta_{i}>\varepsilon\right\}$. Since

$$
0 \leq \frac{(\nu+\lambda)|\lambda-1|}{\left(\nu_{q}+1\right)\left(\lambda-1+\sum_{i=0}^{\nu_{q}} \beta_{i, n}\right)} \leq \frac{(\nu+\lambda)|\lambda-1|}{\left(\nu_{q}+1\right)\left(\lambda-1+\sum_{i=0}^{\nu_{q}} \beta_{i}\right)} \leq 1+\mu
$$

we have that

$$
\begin{aligned}
& \lim _{n \rightarrow \infty} \sup _{\lambda \in[a, b]} \mathbb{E}\left[\frac{(\nu+\lambda)|\lambda-1| \mathbf{1}_{\mathcal{B}_{\varepsilon}^{c}}}{\left(\nu_{q}+1\right)\left(\lambda-1+\sum_{i=0}^{\nu_{q}} \beta_{i}\right)}-\frac{(\nu+\lambda)|\lambda-1| \mathbf{1}_{\mathcal{B}_{\varepsilon}^{c}}}{\left(\nu_{q}+1\right)\left(\lambda-1+\sum_{i=0}^{\nu_{q}} \beta_{i, n}\right)}\right] \\
& \leq(1+\mu) \sup _{\lambda \in[a, b]} \mathbb{P}\left(\mathcal{B}_{\varepsilon}^{c}\right) .
\end{aligned}
$$

Note that $\nu \geq 1$ therefore $\mathbb{P}\left(\mathcal{B}_{\varepsilon}^{c}\right) \leq \mathbb{P}\left(P_{\lambda}^{\mathbf{T}^{*}}\left(\sigma_{e^{*}}=\infty\right) \leq \varepsilon\right) \leq \mathbb{P}\left(P_{b}^{\mathbf{T}^{*}}\left(\sigma_{e^{*}}=\infty\right) \leq \varepsilon\right)$ for all $\lambda \leq b$ since $P_{\lambda}^{\mathbf{T}^{*}}\left(\sigma_{e^{*}}=\infty\right)$ is monotonically decreasing in $\lambda$. Since $b<\mu$ we have that the walk with bias $b$ is $\mathbb{P}$-a.s. transient therefore $\lim _{\varepsilon \rightarrow 0} \mathbb{P}\left(P_{b}^{\mathbf{T}^{*}}\left(\sigma_{e^{*}}=\infty\right) \leq \varepsilon\right)=0$. It follows that $\sup _{\lambda \in[a, b]} \mathbb{P}\left(\mathcal{B}_{\varepsilon}^{c}\right)$ can be made arbitrarily small by choosing $\varepsilon>0$ sufficiently small.

Recalling that $1-\lambda \leq \beta_{i} \leq \beta_{i, n}$, we have that

$$
\begin{aligned}
& \lim _{n \rightarrow \infty} \sup _{\lambda \in[a, b]} \mathbb{E}\left[\frac{(\nu+\lambda)|\lambda-1| \mathbf{1}_{\mathcal{B}_{\varepsilon}}}{\left(\nu_{q}+1\right)\left(\lambda-1+\sum_{i=0}^{\nu_{q}} \beta_{i}\right)}-\frac{(\nu+\lambda)|\lambda-1| \mathbf{1}_{\mathcal{B}_{\varepsilon}}}{\left(\nu_{q}+1\right)\left(\lambda-1+\sum_{i=0}^{\nu_{q}} \beta_{i, n}\right)}\right] \\
& =\lim _{n \rightarrow \infty} \sup _{\lambda \in[a, b]} \mathbb{E}\left[\frac{(\nu+\lambda)|\lambda-1|}{\nu+1} \frac{\mathbf{1}_{\mathcal{B}_{\varepsilon}} \sum_{i=0}^{\nu_{q}}\left(\beta_{i, n}-\beta_{i}\right)}{\left(\lambda-1+\sum_{i=0}^{\nu_{q}} \beta_{i}\right)\left(\lambda-1+\sum_{i=0}^{\nu_{q}} \beta_{i, n}\right)}\right] \\
& \leq \mu(\mu+1) \lim _{n \rightarrow \infty} \sup _{\lambda \in[a, b]} \mathbb{E}\left[\frac{\mathbf{1}_{\mathcal{B}_{\varepsilon}} \sum_{i=0}^{\nu_{q}}\left(\beta_{i, n}-\beta_{i}\right)}{\left(\lambda-1+\sum_{i=0}^{\nu_{q}} \beta_{i}\right)^{2}}\right] \\
& \leq \frac{\mu(\mu+1)}{\varepsilon^{2}} \lim _{n \rightarrow \infty} \sup _{\lambda \in[a, b]} \mathbb{E}\left[\sum_{i=0}^{\nu_{q}}\left(\beta_{i, n}-\beta_{i}\right)\right] \\
& \leq \frac{\mu(\mu+1) \mathbb{E}[\nu+1]}{\varepsilon^{2}} \lim _{n \rightarrow \infty} \sup _{\lambda \in[a, b]} \mathbb{E}\left[P_{\lambda}^{\mathbf{T}^{*}}\left(n<\sigma_{e^{*}}<\infty\right)\right]
\end{aligned}
$$

which is equal to 0 by (2.4).

We now extend to the setting where $p_{0} \neq 0$. By independence of $\left(\nu, \nu_{q}\right)$ with $\left(\beta_{i}, \beta_{i, n}\right)_{i \geq 0}$ we have that

$$
\begin{aligned}
& \mathbb{E}\left[\frac{(\nu+\lambda)|\lambda-1|}{\left(\nu_{q}+1\right)\left(\lambda-1+\sum_{i=0}^{\nu_{q}} \beta_{i}\right)}-\frac{(\nu+\lambda)|\lambda-1|}{\left(\nu_{q}+1\right)\left(\lambda-1+\sum_{i=0}^{\nu_{q}} \beta_{i, n}\right)}\right] \\
& =\mathbb{E}\left[\frac{(\nu+\lambda)|\lambda-1|}{\left(\nu_{q}+1\right)}\left(\frac{1}{\lambda-1+\sum_{i=0}^{\nu_{q}} \beta_{i}}-\frac{1}{\lambda-1+\sum_{i=0}^{\nu_{q}} \beta_{i, n}}\right)\right] \\
& =\mathbb{E}\left[\frac{(\nu+\lambda)|\lambda-1|}{\left(\nu_{q}+1\right)} \mathbb{E}\left[\frac{\sum_{i=0}^{\nu_{q}}\left(\beta_{i, n}-\beta_{i}\right)}{\left(\lambda-1+\sum_{i=0}^{\nu_{q}} \beta_{i}\right)\left(\lambda-1+\sum_{i=0}^{\nu_{q}} \beta_{i, n}\right)} \mid \nu_{q}\right]\right] \\
& \leq \mathbb{E}\left[\frac{(\nu+\lambda)|\lambda-1|}{\left(\nu_{q}+1\right)} \sum_{i=0}^{\nu_{q}} \mathbb{E}\left[\frac{\left(\beta_{i, n}-\beta_{i}\right)}{\left(\lambda-1+\beta_{i}\right)\left(\lambda-1+\beta_{i, n}\right)} \mid \nu_{q}\right]\right] \\
& =\mathbb{E}\left[(\nu+\lambda)|\lambda-1|\left(\frac{1}{\lambda-1+\beta_{0}}-\frac{1}{\lambda-1+\beta_{0, n}}\right)\right] \\
& =\mathbb{E}[\nu+\lambda] \mathbb{E}\left[\frac{|\lambda-1|}{\lambda-1+\beta_{0}}-\frac{|\lambda-1|}{\lambda-1+\beta_{0, n}}\right]
\end{aligned}
$$

where we have used that the pairs $\left(\beta_{i}, \beta_{i, n}\right)$ are identically distributed for $i \geq 0$. It therefore suffices to show that

$$
\lim _{n \rightarrow \infty} \sup _{\lambda \in[a, b]} \mathbb{E}\left[\frac{|\lambda-1|}{\lambda-1+\beta_{0}}-\frac{|\lambda-1|}{\lambda-1+\beta_{0, n}}\right]=0 .
$$


The speed of a biased random walk on a Galton-Watson tree is analytic have

Fix $p \in\left(1, \log \left(\lambda_{c}\right) / \log (a)\right)$ and let $q=p /(p-1)$. For $\varepsilon>0$, by Hölder's inequality we

$$
\begin{aligned}
& \sup _{\lambda \in[a, b]} \mathbb{E}\left[\frac{|\lambda-1|}{\lambda-1+\beta_{0}}-\frac{|\lambda-1|}{\lambda-1+\beta_{0, n}}\right] \\
& \leq \varepsilon+\sup _{\lambda \in[a, b]} \mathbb{E}\left[\left(\frac{|\lambda-1|}{\lambda-1+\beta_{0}}-\frac{|\lambda-1|}{\lambda-1+\beta_{0, n}}\right) \mathbf{1}_{\frac{|\lambda-1|}{\lambda-1+\beta_{0}}-\frac{|\lambda-1|}{\lambda-1+\beta_{0, n}}>\varepsilon}\right] \\
& \leq \varepsilon+\sup _{\lambda \in[a, b]} \mathbb{E}\left[\left(\frac{|\lambda-1|}{\lambda-1+\beta_{0}}\right)^{p}\right]^{1 / p} \mathbb{P}\left(\frac{|\lambda-1|}{\lambda-1+\beta_{0}}-\frac{|\lambda-1|}{\lambda-1+\beta_{0, n}}>\varepsilon\right)^{1 / q} .
\end{aligned}
$$

The result then follows from Lemmas 2.1 and 2.2.

\section{References}

[1] E. Aïdékon, Note on the monotonicity of the speed of the biased random walk on a GaltonWatson tree, Unpublished note, 2013. MR3230003

[2] E. Aïdékon, Speed of the biased random walk on a Galton-Watson tree, Probab. Theory Related Fields 159 (2014), no. 3-4, 597-617. MR3230003

[3] K. Athreya and P. Ney, Branching processes, Dover Publications, Inc., Mineola, NY, 2004. MR2047480

[4] M. Barlow, Random walks and heat kernels on graphs, Cambridge University Press, Cambridge, 2017. MR3616731

[5] G. Ben Arous and A. Fribergh, Biased random walks on random graphs, Probability and statistical physics in St. Petersburg, Proc. Sympos. Pure Math., vol. 91, Amer. Math. Soc., Providence, RI, 2016, pp. 99-153. MR3526827

[6] G. Ben Arous, A. Fribergh, N. Gantert, and A. Hammond, Biased random walks on GaltonWatson trees with leaves, Ann. Probab. 40 (2012), no. 1, 280-338. MR2917774

[7] G. Ben Arous, A. Fribergh, and V. Sidoravicius, Lyons-Pemantle-Peres monotonicity problem for high biases, Comm. Pure Appl. Math. 67 (2014), no. 4, 519-530. MR3168120

[8] G. Ben Arous, Y. Hu, S. Olla, and O. Zeitouni, Einstein relation for biased random walk on Galton-Watson trees, Ann. Inst. Henri Poincaré Probab. Stat. 49 (2013), no. 3, 698-721. MR3112431

[9] A. Bowditch, Escape regimes of biased random walks on Galton-Watson trees, Probab. Theory Related Fields 170 (2018), no. 3-4, 685-768. MR3773798

[10] A. Bowditch, A quenched central limit theorem for biased random walks on supercritical Galton-Watson trees, J. Appl. Probab. 55 (2018), no. 2. MR3832907

[11] A. Bowditch, Central limit theorems for biased randomly trapped walks on $\mathbb{Z}$, Stochastic Process. Appl. 129 (2019), no. 3. MR3913266

[12] A. Bowditch and Y. Tokushige, Differentiability of the speed of biased random walks on GaltonWatson trees, ALEA Lat. Am. J. Probab. Math. Stat. 17 (2020), no. 1, 609-642. MR4130577

[13] A. Dembo, N. Gantert, Y. Peres, and O. Zeitouni, Large deviations for random walks on Galton-Watson trees: averaging and uncertainty, Probab. Theory Related Fields 122 (2002), no. 2, 241-288. MR1894069

[14] A. Fribergh and A. Hammond, Phase transition for the speed of the biased random walk on the supercritical percolation cluster, Comm. Pure Appl. Math. 67 (2014), no. 2, 173-245. MR3149843

[15] S. Janson, Simply generated trees, conditioned Galton-Watson trees, random allocations and condensation, Probab. Surv. 9 (2012), 103-252. MR2908619

[16] R. Lyons, R. Pemantle, and Y. Peres, Biased random walks on Galton-Watson trees, Probab. Theory Related Fields 106 (1996), no. 2, 249-264. MR1410689

[17] S. Mehrdad, B. Sen and L. Zhu, The speed of a biased walk on a Galton-Watson tree without leaves is monotonic with respect to progeny distributions for high values of bias, Ann. Inst. Henri Poincaré Probab. Stat. 51 (2015), no. 1, 304-318. MR3300972 
The speed of a biased random walk on a Galton-Watson tree is analytic

[18] Y. Peres and O. Zeitouni, A central limit theorem for biased random walks on Galton-Watson trees, Probab. Theory Related Fields 140 (2008), no. 3-4, 595-629. MR2365486

[19] A. Sznitman, Slowdown estimates and central limit theorem for random walks in random environment, J. Eur. Math. Soc. (JEMS) 2 (2000), no. 2, 93-143. MR1763302

Acknowledgments. We would like to thank the anonymous referee for a thorough examination of the preliminary version and some helpful comments. A. B. acknowledges support of NUS grant R-146-000-260-114. Y. T. was supported by JSPS KAKENHI Grant Number JP16H06338.

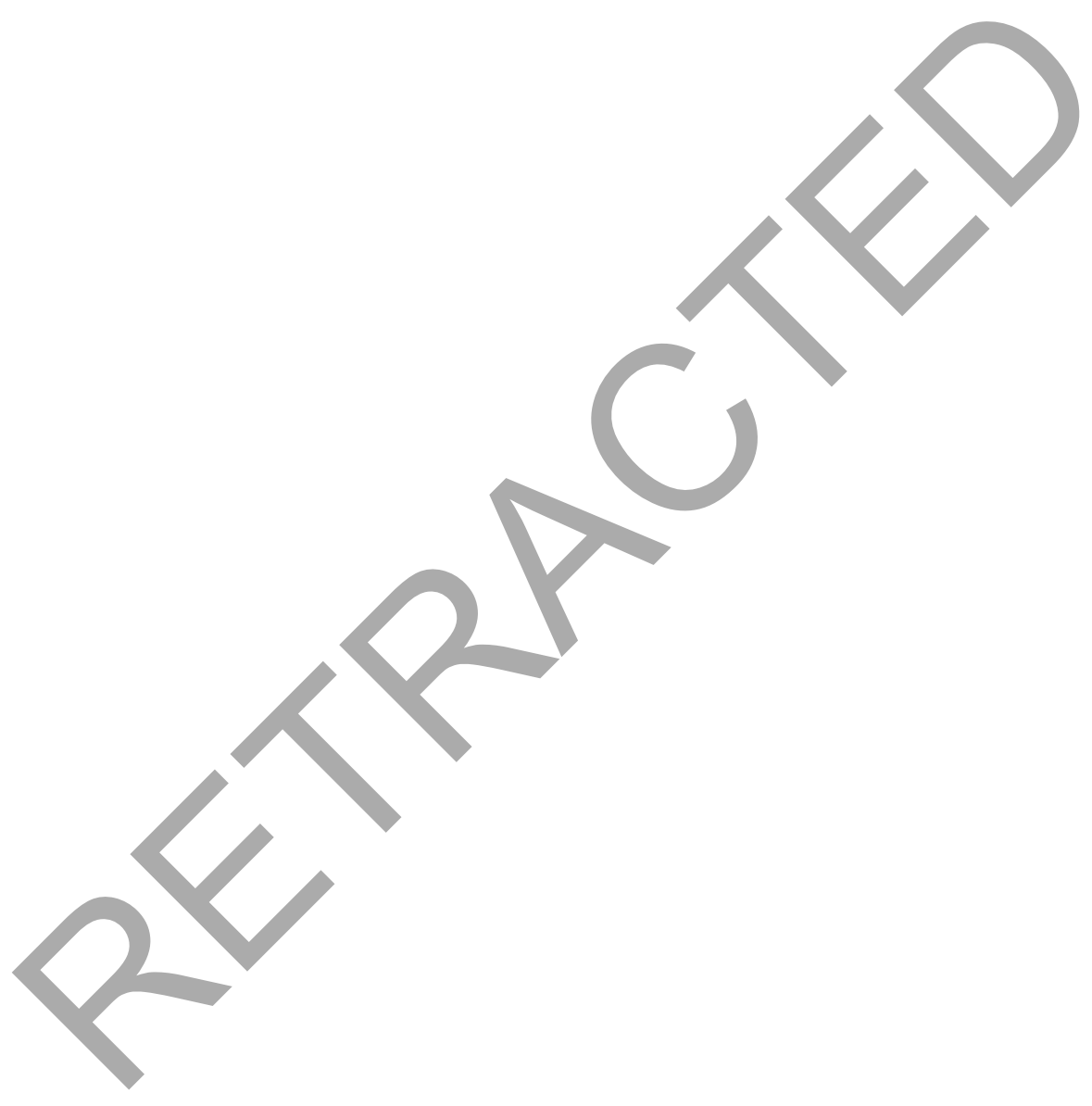




\section{Electronic Journal of Probability Electronic Communications in Probability}

\section{Advantages of publishing in EJP-ECP}

- Very high standards

- Free for authors, free for readers

- Quick publication (no backlog)

- Secure publication $\left(\mathrm{LOCKSS}^{1}\right)$

- Easy interface (EJMS²)

Economical model of EJP-ECP

- Non profit, sponsored by $\mathrm{MSS}^{3}, \mathrm{BS}^{4}$, ProjectEuclid ${ }^{5}$

- Purely electronic

\section{Help keep the joungal free and vigorous}

- Donate to the IMS open access fund ${ }^{6}$ (click here to donate!)

- Submit your best articles to EJP-ECP

- Choose EJP-ECP over for-profit journals

\footnotetext{
${ }^{1}$ LOCKSS: Lots of Copies Keep Stuff Safe http://www . lockss.org/

${ }^{2}$ EJMS: Electronic Journal Management System http://www.vtex.lt/en/ejms.html

${ }^{3}$ IMS: Institute of Mathematical Statistics http://www.imstat.org/

${ }^{4}$ BS: Bernoulli Society http://www . bernoulli-society.org/

${ }^{5}$ Project Euclid: https://projecteuclid.org/

${ }^{6}$ IMS Open Access Fund: http://www.imstat.org/publications/open.htm
} 